\title{
Influence of admixture atom chemosorption on properties of p-electron conjugated system of open carbon nanotubes
}

\author{
O. B. Tomilin, E. V. Rodionova, E. A. Rodin, E. E. Muryumin \\ N. P. Ogarev National Research Mordovian State University, Saransk, Russia \\ tomilinob@mail.ru, rodionova_j87@mail.ru, evg.rodin54@gmail.com,mur_ee@mail.ru
}

PACS 61.46. $+w$, 61.48.De, 68.43.-h, 79.70. $+q$

DOI 10.17586/2220-8054-2018-9-1-70-72

Basing on the hypothesis of the emission molecular orbitals (EMO) existence in single-walled carbon nanotubes generated by in-plane-electron conjugation of $p$-electrons, we studied influences of adsorbate $\left(\mathrm{H}_{2}\right.$ and $\left.\mathrm{F}_{2}\right)$ nature on characteristics of electrons field emission from open single-walled ultrashort carbon nanotubes of chirality $(n, 0)$. It has been shown that the adsorption of admixture atoms on the graphene surface increases the work function of the electron, moreover more considerable value of work function corresponds to more considerable electronegativity of the chemisorption atom.

Keywords: carbon nanotube, p-electron conjugated system, chemosorption atoms, hydrogen, fluorine.

Received: 22 June 2017

Revised: 8 October 2017

\section{Introduction}

At present, carbon nanotubes (CNTs) confidently expand the field of their practical application. Unique mechanical, electro-physical properties, high chemical and thermal stability of CNTs make it possible to use them as part of modern vacuum electronic devices. The most widely used cold field emitters are based on CNTs. Similar cathode materials are used in flat panel displays with increased luminescence brightness, cathode tubes, $\mathrm{X}$-ray sources and other devices [1].

Fowler-Nordheim (FN) theory is used to describe emission of electrons from CNTs under the action of a uniform constant electric field. Initially, this theory was developed for metallic flat emitters [2]. Specific features of the CNTs structure lead to the introduction of different correction factors in various modifications of this theory [3]. However, the fundamental position of the FN theory - the tunneling effect is in no way connected with the characteristics of the real structure of CNTs, which is a deterrent theoretical factor with a purposeful improvement of the operational properties of real cathode materials.

The electron concentration at the ends of CNTs under field emission was experimentally recorded by electron spectroscopy methods, as the field emission process is accompanied in many cases by luminescence [4]. Thus, a non-controversial theory of electron emission from a CNT should proceed from the existence of a molecular state in which the electron density will be mainly localized at the ends of nanotubes.

Papers [5,6] show that in cylindrical carbon molecules due to in-plane electron conjugation of $p$-electrons [7] are formed of emission molecular orbitals (EMO) with extreme localization electrons on the ends of open singlewalled CNT (SWCNT) with chirality $(n, 0)$ and $(n, n)$, are formed, as shown in Fig. 1. The resulting EMO are vacant. Despite the fact that the electric field is not taken into account, the study of EMO characteristic and the influence on them factors of real structure CNT are important for qualitative estimating the parameters of field emission of electrons from the SWCNT.

CNT exhibit the ability to adsorb various low molecular weight gaseous substances $\left(\mathrm{H}_{2}, \mathrm{CO}_{2}\right.$, etc. $)$ [8]. Adsorption of molecules on the graphene surface of CNTs leads to a change in the nanotubes' electronic and, consequently, emission characteristics of nanotubes.

\section{Model and method}

In this paper, we investigated the effect of chemisorption of $\mathrm{H}_{2}$ and $\mathrm{F}_{2}$ molecules on the surface of open ultrashort SWCNT (usSWCNT) of chirality $(n, 0)$ at $n=5-9$ on the properties of the conjugate system of $p$-electrons. The model molecules studied were formed by the consecutive combination of cyclic trans-carbon chains, located on a cylindrical surface usSWCNT symmetrically cylindrical axis of the nanotube. The number of chains, $i$, was varied from $2-10$.

Calculations of the electronic structure of model molecules were carried out by the non-empirical Hartree-Fock (HF) method in the 3-21G basis from the FireFly application package with complete geometry optimization [9]. 


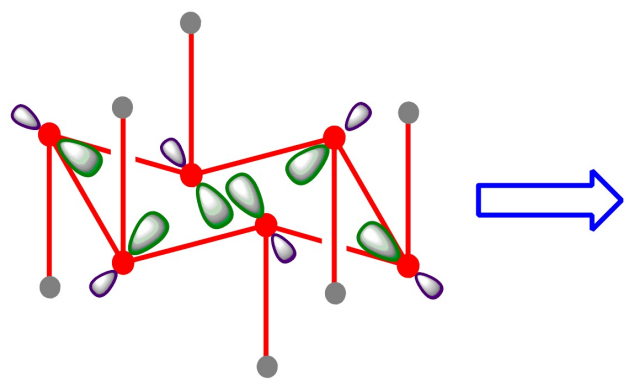

A

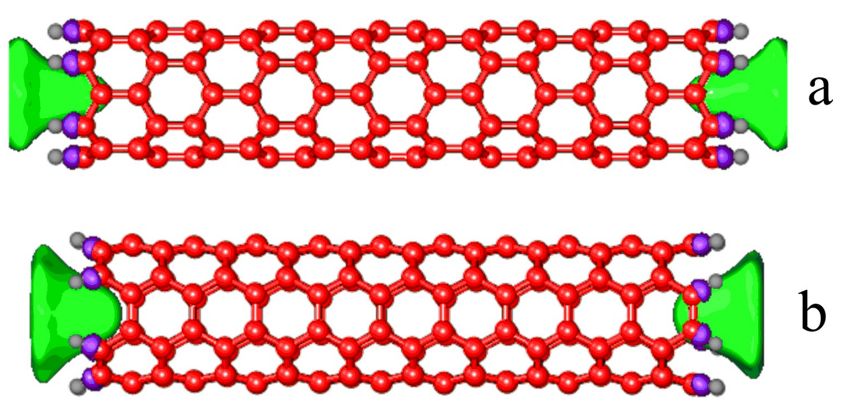

$\mathrm{B}$

FIG. 1. A) Orientation of $p$-orbitals of carbon atoms in monocyclic hydrocarbons CNHN in in-plane conjugation; B) Realization of emission molecular orbitals with in-plane-electron conjugation in carbon nanotubes: a - SWCNT $(6,0), b-\operatorname{SWCNT}(4,4)[4]$

The arrangement of $\mathrm{H}_{2}$ and $\mathrm{F}_{2}$ molecules on the graphene surface of model molecules was carried out randomly with the help of a random number generator, while maintaining the approximate value of the ratio of the number of chemosorption atoms to the total number of carbon atoms in molecule, which was 1/15.

The possibility of $\mathrm{H}_{2}$ and $\mathrm{F}_{2}$ chemisorption for the model molecules was estimated from the value of the adsorption energy of $H / F$ atoms calculated by the formula (1):

$$
E_{a d}=\left(E^{u s S W N T+A}-E^{u s S W N T}-N E^{A}\right) / N,
$$

where $E^{u s S W N T+A}$ is the total energy of an optimized model molecule with adsorbed atoms; $E^{u s S W N T}$ is the total energy of an optimized model molecule without admix atoms, $E^{A}$ is the total energy of free adatoms; $N$ is the number of adatoms in the model molecule. The negative values of $E_{a d}$ indicate on the realization of chemisorption in all usSWCNTs irrespective of their length and diameter.

Analysis of the structure of molecular orbitals in model molecules with adsorbed $H / F$ atoms showed the presence of two emission MOs (Fig. 2). As well as for "pure" opened usSWCNTs the emission orbitals have a vacant character.

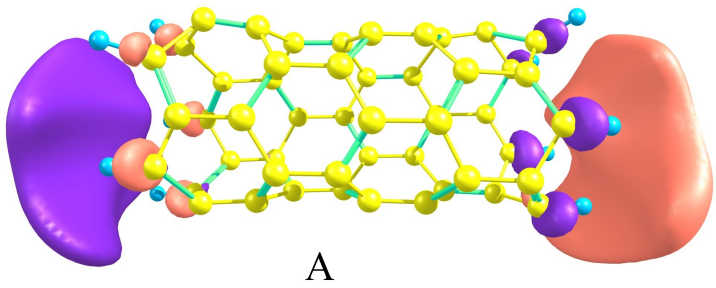

A

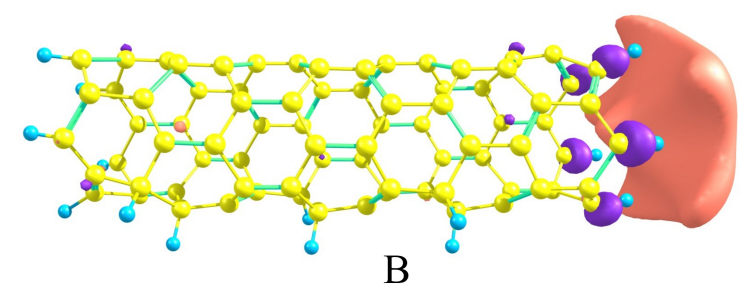

$\mathrm{B}$

FIG. 2. Structure of EMO: A) "pure" opened usSWCNTs; B) opened usSWCNTs with chemisorbed atoms

For all model molecules, the EMO energies tend to one asymptotic limit with an increasing number of cyclic trans-carbon chains, turning into a doubly degenerate state. Previously, a similar result was shown for EMO in "clean" opened usSWCNTs [5].

The energy of the EMO $\left(E^{E M O}\right)$ at $i=10$ decreases monotonically with increasing chirality index $n$. The values of $E^{E M O}$ in the model molecules depend on the electronegativity of the chemisorbed atoms: $E^{E M O}(H)<E^{E M O}$ ("pure") $<E^{E M O}(F)$.

The work function of the CNT's electron can be qualitatively compared with the value of $\Delta E$, equal to $\Delta E=E^{E M O}-E^{H O M O}$, where HOMO is the highest occupied molecular orbital.

$\Delta E$ is an estimate of the transition process energy of the $p$-electrons conjugated system from the delocalization state along the carbon core to the state with localization of the electron density at the ends of the carbon nanotube. This supposition is in correspondence with the stepwise emission process of the FN theory, as well as the available experimental data [4]. 
Figure 3 shows the graphical dependencies of $\Delta E$ on the chirality index $n$ in model molecules at $i=10$ for "pure" usSWCNT and usSWCNT with chemisorbed atoms on the graphene surface. Fig. 3 shows that the value of $\Delta E$ for usSWCNTs $(n, 0)$ with chemisorbed atoms is greater than the value of $\Delta E$ for "pure" usSWCNTs. Moreover, for the chemisorption of fluorine atoms, the value of $\Delta E$ is greater than in the case of chemisorption of hydrogen atoms.

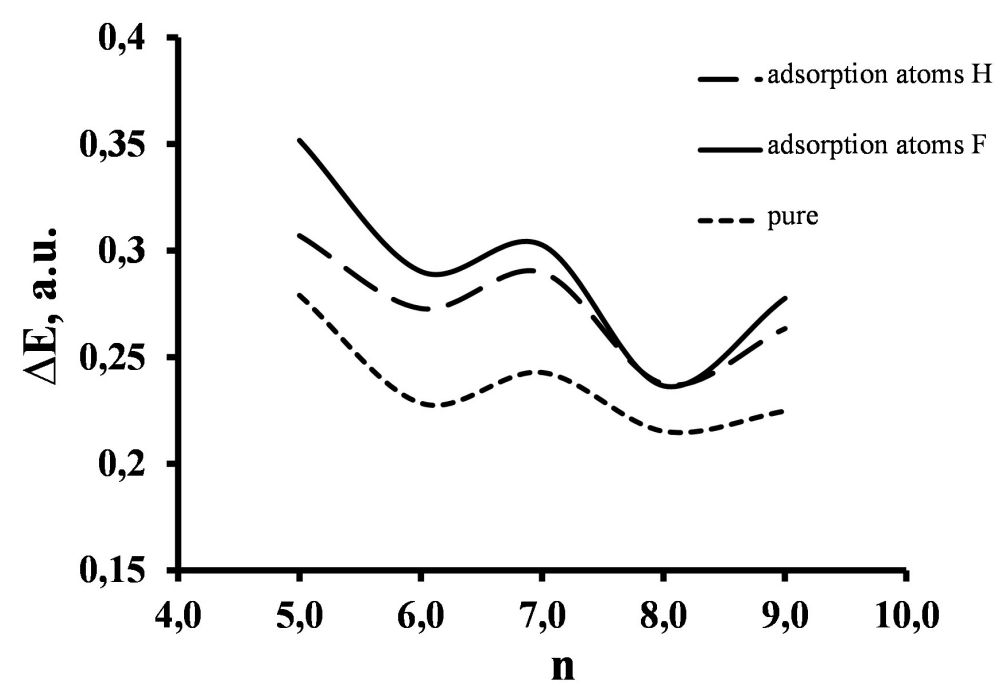

FIG. 3. Dependence of $\Delta E$ on the chirality index $n$ in usSWCNTs $(n, 0)$ with the number of cyclic trans-carbon chains $i=10$

The results of the research proved that the population of the emission state in $\operatorname{SWCNT}(n, 0)$ with chemisorbed atoms is less than in "pure" carbon nanotubes, so adsorption of gases on the graphene surface of a CNT should lead to a decrease in the emission current density. This result corresponds to the experimental data of [10], where it is shown that annealing of carbon nanotubes (removal of impurity atoms from a graphene surface) leads to an increase in the density of the emission current.

\section{References}

[1] Rakov E.G. Carbon nanotubes in new materials. Russian Chem. Rev., 2013, 82 (1), P. $27-47$.

[2] Fowler R.H., Nordheim L. Electron emission in intense electric fields. Proc. Roy. Soc. London. A, 1928,119 (781), P. $173-181$.

[3] Eletskii A.V. Carbon nanotube-based electron field emitters. Physics - Uspekhi, 2010, 53, P. 863-892.

[4] Bonard J.-M., Stockli T., et al. Field-emission-induced luminescence from carbon nanotubes. Phys. Rev. Lett., 1998,81 (7), P. 1441-1444.

[5] Tomilin O.B., Rodionova E.V., Muryumin E.E., Rodin E.A. Mechanism of field emission of electrons in carbon nanotubes. Proc. 5th Intern. Conf. on Nanostructured Materials2016, Minsk, Belarus, November 22-25, 2016, Belarusian Science, 400 p.

[6] Tomilin O.B., Stankevich I.V., Muryumin E.E., Rodionova E.V. Electronic conjugation of carbon atoms in spherical and cylindrical molecules. Carbon, 2012, 50, P. 5217-5225.

[7] Schleyer P.v.R., Jiao H., et al. Double Aromaticity in the 3,5-Dehydrophenyl Cation and in Cyclo[6]carbon. J. Amer. Chem. Soc., 1994, 116, P. 10129-10134.

[8] Bauschlicher C.W., Jr. Hydrogen and fluorine binding to the sidewalls of a $(10,0)$ carbon nanotube. Chem. Phys. Lett., 2000, 322, P. 237-241.

[9] Schmidt M.W., Baldridge K.K., et al. General Atomic and Molecular Electronic Structure System. J. Comput. Chem., 1993, 14, P. 13471363.

[10] Zhao G., Zhang J., et al. Fabrication and characterization of single carbon nanotube emitters as point electron sources. Appl. Phys. Lett., 2006, 89, 193113(3). 